\title{
Actividad física y enfermedad
}

\author{
J. L. ESCOLAR CASTELLÓN, C. PÉREZ ROMERO DE LA CRUZ', R. CORRALES \\ MÁRQUEZ
}

Servicios de Medicina Interna y de ${ }^{1}$ Fisioterapia y Rehabilitación. Hospital Universitario Virgen de la Victoria. Málaga

\author{
PHYSICAL ACTIVITY AND DISEASE
}

\section{RESUMEN}

El sedentarismo es hoy día muy frecuente en nuestra sociedad y constituye un factor de riesgo para una amplia patología. La actividad física proporciona unos efectos beneficiosos múltiples que disminuyen la morbi-mortalidad general. Se establecen las diferencias entre ejercicio, actividad y forma física. Se comentan las particularidades fisiopatológicas de los ejercicios aerobio y anaerobio y sus beneficios sobre la salud. Se enumeran los principales procedimientos de medida de la actividad física: calorimetría, METS, cuentapasos, pulsómetros y encuestas-cuestionario. Se describen referencias prácticas para llevar a cabo un actividad física suficiente en la vida diaria.

PALABRAS CLAVE: Actividad física y enfermedad.

\begin{abstract}
The sedentarism is a frecuent situation in our modern society, and represents a risk factor for a vast array of pathology. The physical activity produces several positive effects, which decreases the general morbi-mortality of the population. There is made a commentary about some definitions: Exercise, activity and physical form, aerobic and anaerobic work, muscle exercise. Their pathophysiology and their health benefits. We described the measurement procedures: calorimetry, oxigen consum, accelerometers, pulsometers and cuestionnaries. There is finally done a review upon practical norms.
\end{abstract}

KEY WORDS: Physical activity and disease.

Escolar Castellón JL, Pérez Romero de la Cruz C, Corrales Márquez. R. Actividad física y enfermedad. An Med Interna (Madrid) 2003; 20: 427-433

\section{INTRODUCCIÓN}

La sociedad actual se caracteriza por propiciar un incremento de la automatización y de la mecanización del trabajo físico, lo que, junto a una mayor oferta alimentaria, ha supuesto un innegable bienestar para la población. Sin embargo, el exceso en la utilización de estos medios, en principio beneficiosos, lleva a consecuencias que, cómo el sedentarismo y el exceso nutricional, representan en nuestros días una amenaza para la salud de las personas. Constituyéndose así en factores de riesgo de patologías crónicas muy frecuentes. Lo que ha obligado a consensuar mensajes de salud pública sobre medidas higiénico-sanitarias, tanto en cuestiones alimentarias como de actividad física (1-3). El problema alimenticio se conoce desde hace tiempo y se ha estudiado en profundidad, no sólo en sus facetas preventivas, sino también curativas, por lo que se dispone de amplias bases científicas al respecto.
En los últimos años, está tomando gran relevancia en Medicina, la necesidad de realizar trabajo físico y su consideración como agente de salud. Mediante estudios controlados, de carácter epidemiológico, clínico y experimental se han constatado los efectos beneficiosos ejercidos por la práctica de la actividad física, al observar la disminución que se produce tanto en la frecuencia de aparición, cómo en la rapidez de progresión de muchas enfermedades prevalentes, entre las que se destacan: La insuficiencia coronaria (4-7), la insuficiencia cardiaca $(8,9)$, la hipertensión arterial (10), el ictus cerebral (11), la diabetes mellitus tipo 2 (12), la osteoporosis $(13,14)$ y el cáncer de colon $(15,16)$. También se ha comprobado una menor incidencia de los llamados factores de riesgo cardiovascular, cómo son el perfil lipídico (17-25), la presión arterial, la tolerancia a la glucosa $(26,27)$ o la sensibilidad a la insulina (28). Es llamativa la modulación positiva sobre las funciones orgánicas generales, cómo son la inflamación

Trabajo aceptado: 31 de marzo de 2003

Correspondencia: José Luis Escolar Castellón. Servicio de Medicina Interna. Hospital Virgen de la Victoria. Campus Universitario de Teatinos. 29010 Málaga. 
$(29,9)$, la inmunidad $(8,30)$ o la distribución de los depósitos grasos (31). Y también se han apreciado mejorías de las funciones psíquicas, consistentes en un mayor optimismo (32), una menor ansiedad (33) y una mejora del sueño nocturno. Todos estos cambios propician, a cualquier edad, una mayor calidad de vida y disminuyen la morbi-mortalidad, no sólo cardiovascular, sino también global (34).

En países socio-económicamente avanzados solamente el tercio de la población adulta realiza actividad física suficiente, es decir, que cumple con los criterios aconsejados por las autoridades sanitarias. Por el contrario, los dos tercios restantes, o llevan una vida claramente sedentaria o desarrollan un trabajo físico a todas luces insuficiente. Los estudios epidemiológicos realizados en nuestro país, revelan hechos similares a los observados en otros de igual nivel social, respeto a la presencia de factores de riesgo que condicionan el sedentarismo. Éste se asocia preferentemente al sexo femenino (35), al hábito tabáquico, al aumento de la edad (36) y a niveles económicos y educacionales bajos. Además, hay que añadir otros factores, cómo son: la falta de tiempo, el exceso de peso, el sedentarismo ambiental, tanto en familiares, como en amigos y en personal sanitario, la ausencia de instalaciones abiertas que propicien ejercicio (carriles y caminos destinados a pasear, correr o montar en bicicleta), el mal tiempo habitual, la inseguridad ciudadana, el exceso de televisión, etc. (37-39).

\section{BASES FISIOPATOLÓGICAS}

Se denomina actividad física a cualquier movimiento o aplicación de fuerza del músculo esquelético que resulta en un gasto de energía. A la actividad física planificada, estructurada y repetitiva, realizada para mantener o mejorar una forma física se le llama ejercicio físico. Se entiende por forma física al conjunto de capacidades de la persona que le permite realizar una actividad física, sin que aparezcan molestias, cómo pueden ser entre otros, los síntomas de fatiga, disnea o agotamiento.

El ejercicio físico determina cambios fisiológicos consistentes en un incremento del consumo de energía y una activación de la circulación sanguínea. El sistema nervioso vegetativo, de predominio simpático interviene en el proceso. Se conoce la existencia de centros en el hipotálamo posterior en donde existen diversas sustancias neurotransmisoras, que regulan las respuestas cardiorrespiratorias al ejercicio físico, cómo el ácido gamma aminobutírico (GAB) y sus enzimas, entre ellas la descarboxilasa del ácido glutámico (GAD) (40).

El trabajo muscular puede ser de dos tipos: aerobio y anaerobio. Se denomina ejercicio aerobio al que tiene cómo resultado el movimiento, es isotónico, sin que se desarrolle al mismo tiempo una fuerza excesiva, como sería el producido al andar o correr. Se llama aerobio por que consume oxígeno para la producción de energía. Puede ser desarrollado durante largos periodos de tiempo. Lleva aparejada la proliferación de fibras musculares tipo 2, de contracción lenta. Por el contrario, se denomina ejercicio anaerobio el que promueve la realización de fuerza, con poco o ningún movimiento muscular. La anaerobiosis se realiza durante los llamados ejercicios isométricos (utilización de pesas y aparatos de gimnasio, etc.). Este tipo de trabajo estimula el desarrollo de las fibras musculares tipo 1 de despolarización rápida. Podemos considerar anaeróbicos a los llamados ejercicios calisténicos, desarrollo de fuer- za utilizando el propio cuerpo ("fondo de brazos", es decir, flexión de brazos en el suelo, etc.). En el trabajo anaeróbico, la contracción mantenida del músculo impide el aporte sanguíneo, la energía se obtiene del metabolismo anaerobio. Los recursos energéticos son aprovechados de forma menos eficaz que en el ejercicio aeróbico. Va acompañado de la producción de catabolitos, como el ácido láctico. Es de obligada corta duración, pudiéndose mantener sólo unos pocos minutos. Una vez terminado el ejercicio anaerobio se restituye en los músculos el almacén de los sustratos que se habían consumido. Sin embargo, durante los tres primeros minutos de un ejercicio aeróbico y hasta que el sistema vegetativo pone en marcha la adaptación orgánica global, el metabolismo muscular es anaerobio (sprinters), lo mismo ocurre en los casos en los que el ejercicio se fuerza más alla de la forma física del sujeto (agotamiento de los ciclistas).

El trabajo muscular desencadena directamente, sea cual sea su forma, aeróbica o anaeróbica, un consumo de energía y unos cambios metabólicos beneficiosos, entre los que se destacan:

1. Disminución de los depósitos grasos, al potenciar la acción de las medidas dietéticas. Favorece la pérdida de peso y el mantenimiento del peso alcanzado. El ejercicio físico ayuda además a normalizar la sensación fisiológica de hambre y saciedad (41-45). Para obtener una pérdida de peso importante a base de ejercicio físico, se tendrían que realizar, bien deportes de alta competición o bien trabajos muy intensos y mantenidos, lo que en la vida actual es difícil de conseguir fuera del profesionalismo deportivo. El ejercicio físico además, es necesario para controlar las pérdidas de peso y la redistribución de tejido adiposo en situaciones lipodistróficas, cómo son algunos tratamientos anti-VIH (46), el correspondiente a las mujeres postmenopáusicas $(47,48)$ y el acompañante al síndrome de Cushing.

2. Aumento de receptores de insulina y lipoproteínas (49), con la mejoría de los perfiles glucídicos y lipémicos.

3. Producción de citoquinas y prostaglandinas que regulan favorablemente mecanismos inmunitarios (50) y de la hemostasia (51), con acción antiinflamatoria y antitrombótica, al intervenir en la fibrinolisis y función plaquetaria.

4. Aumento de los antioxidantes (52).

5. Secreción de endorfinas con efectos neuropsicológicos, llevando a una disminución de la ansiedad y depresión, menor percepción del dolor, mejora la afectividad y las relaciones sociales, mejora el sueño, etc.

6. La acción sobre el aparato cardio-vascular, lleva a respuestas diferentes según el tipo de ejercicio. El ejercicio aeróbico tiene un efecto tonificador beneficioso, al desencadenar la vasodilatación periférica y aumentar el volumen minuto, disminuyendo a la larga la tensión arterial y mejorando el funcionalismo cardiaco. El ejercicio anaerobio, que produce un aumento de la masa muscular esquelética y por lo tanto de la fuerza, lleva asociada una vasoconstricción que desencadena elevaciones a veces peligrosas de la presión arterial, con la consiguiente sobrecarga sistólica cardiaca, por lo que no es recomendable a los efectos aquí comentados. Ya la simple contracción de un grupo muscular aislado, como sería el producido al cerrar el puño, desencadena una respuesta orgánica general simpática, con vasoconstricción periférica y elevación de la tensión arterial.

La respuesta a la actividad física, no es igual en toda la población. Esta condicionada por la constitución del indivi- 
duo, por la herencia, es decir por la composición genética (53). Los efectos que se producen, son diferentes entre individuos, pudiendo observarse rasgos comunes dentro de una misma familia (54-57). Existen también algunas diferencias entre sexos. En las mujeres el trabajo físico, en igualdad de condiciones, desencadena una mayor lipolisis que en el varón, en el que predomina la glucogenolisis $(58,59)$.

El tiempo de aparición de la respuesta orgánica al ejercicio físico es variable y los efectos metabólicos son diversos, pudiendo constatarse efectos inmediatos y pasajeros, ya a los pocos minutos, en el caso de los triglicéridos, la tensión arterial o la glucemia. De aquí la necesidad de practicar la actividad física de forma casi diaria. Un efecto más estable se desarrolla a partir de las dos o tres semanas de comenzar a realizar el ejercicio físico. En algunas ocasiones los cambios detectables se inician algo más tarde, cómo es el caso del colesterol HDL, en el que las elevaciones plasmáticas tardan en presentarse hasta 9 meses (60). Se ha observado cómo, ya durante los dos años siguientes al comienzo de la actividad física hay una disminución de la morbi-mortalidad cardiovascular. La duración de los efectos beneficiosos es pasajera, desapareciendo aproximadamente al mes de su interrupción (32).

\section{MÉTODOS DE MEDIDA}

Para la cuantificación del trabajo muscular se dispone hoy día de una serie de procedimientos:

La calorimetría directa. Es el método más clásico, basado en el calor producido por el organismo. Es una forma global de medir la actividad física, se expresa en Kcalorias o en Kjulios. La exploración se realiza dentro de cámaras cerradas, por lo que su aplicación es exclusivamente experimental.

El consumo de oxígeno. Es dependiente del trabajo aeróbi$\mathrm{co}$, y se define en $\mathrm{ml} / \mathrm{seg}$. Al requerir la conexión a una fuente de oxígeno, su empleo se hace fundamentalmente con fines de investigación y para estudios en ejercicios de corta duración.

Con los datos aportados por esas pruebas, se puede calcular de forma indirecta, el consumo de energía realizado. Se dispone a tal fín de tablas y ecuaciones que relacionan el consumo de oxígeno con la intensidad del trabajo, lo que se expresa en METS. El MET es una unidad que cuantifica el esfuerzo físico. Esta unidad se utiliza como referencia en las pruebas de sobrecarga cardiorrespiratoria, como ocurre en el test ergométrico de Bruce (61). Un MET es un equivalente metabólico y corresponde al esfuerzo físico que obliga a un consumo de oxígeno de $3,5 \mathrm{ml} /$ minuto por kg de peso corporal. La intensidad de trabajo de un MET durante una hora, corresponde a la producción de $80 \mathrm{Kcal}$, o de 336 Kjulios (1 $\mathrm{kcal}=4,19 \mathrm{kjul}$ ) y equivale a la intensidad del gasto orgánico realizado al estar en reposo, leyendo, o conduciendo un automóvil. La marcha en llano a la velocidad de $5 \mathrm{~km} /$ hora, el bajar escaleras a 60-70 escalones / minuto, el paseo en bicicleta a $15 \mathrm{~km} /$ hora, suponen una intensidad de 4 METS. La carrera en llano (footing), a $10 \mathrm{~km} /$ hora, supone 9 METS. Las personas sanas sedentarias pueden alcanzar una actividad física de 9-11 METS, mientras que los individuos entrenados llegan a los 16 METS. La ausencia de síntomas cardiológicos a niveles de intensidad iguales o superiores a 5 METS, durante una ergometría, indica un bajo riesgo coronario, lo que en la vida normal permitiría esfuerzos de hasta 8 METS si son de corta duración, por ejemplo, subir tres tramos de escaleras a paso lento.

El ejercicio físico diario de tipo aeróbico se puede calcular de forma aproximada a partir del tipo de movimiento efectuado, especificando el tiempo y el número de días a la semana en el que se practica. Una forma habitual de hacerlo es la de considerar el espacio recorrido en un tiempo (velocidad), al realizar un determinada actividad, cómo sería caminar o correr.

Existen otros procedimientos, de fundamentos más indirectos, pero quizá más idóneos para su aplicación, ya que miden la actividad física realizada durante largos periodos de tiempo, incluso varios días. Son los "cuenta pasos" y se basan en la detección, mediante aparatos portátiles miniaturizados, de la inercia originada en los cambios de postura corporal, provocados al realizar movimientos, generalmente dar pasos (62-66). Se utilizan así los "acelerómetros tridimensionales”, que fijados al cuerpo de la persona registran electrónicamente los movimientos, permitiendo calcular el número de "pasos" dado en un largo periodo de tiempo, generalmente de varios días. Calculadoras incorporadas permiten, conociendo el peso del individuo y su talla, hallar las distancias recorridas o los gastos energéticos efectuados. Más económicos y asequibles son los llamados contadores de pasos o "podómetros" con un mecanismo más simple, basado en el desplazamiento de un péndulo o bola que establece los contactos eléctricos en el interior del aparato. Estudios realizados con estos dos sistemas demuestran una gran fiabilidad y concordancia entre los mismos. Generalmente estos procedimientos se utilizan para medir la actividad de movimiento de forma comparativa, bien frente a valores establecidos para la media de la población o bien frente a las variaciones que se producen en una misma persona a lo largo de un determinado periodo de tiempo.

Para medir la intensidad del esfuerzo y la respuesta orgánica al mismo, se utilizan los "pulsómetros". Son aparatos portátiles provistos de detectores, que colocados, por ejemplo, sobre el tórax o en los pulpejos de los dedos miden la frecuencia de pulso y permiten deducir la forma física del individuo ante un trabajo. Normalmente se recomienda no superar un número de pulsaciones por minuto equivalente a la cifra resultante de restar a 220 la edad del sujeto, debiendo mantener durante el ejercicio una frecuencia cardiaca alrededor del $75 \%$ de dicha cifra. El empleo simultaneo de un contador de pasos y un pulsómetro aporta detalles complementarios, ya que cada uno de estos métodos aisladamente tiene sus limitaciones. Los "cuenta pasos" miden el movimiento, pero infravaloran el esfuerzo muscular, mientras que el pulsómetro, refleja bien la respuesta al esfuerzo orgánico pero detecta peor el total del trabajo realizado $(67,68)$.

En los últimos años se están desarrollando sistemas de cuantificación del movimiento mediante receptores que con un emisor portátil y enlaces vía satélite permiten detectar los desplazamientos de la persona en los tres ejes del espacio, pudiéndose así precisar, al localizarlo, las características del recorrido que el individuo efectúe (tecnología de navegación GPS) $(69,70)$. En la toma de información es también de interés la utilización de los cuestionarios, en los que de una manera retrospectiva, bien de forma autónoma por el sujeto o con la participación de un encuestador, se anotan parámetros referentes al ejercicio realizado en los días previos. Es importante subrayar que el cuestionario debe hacer referencia a varios parámetros de actividad y ejercicio. La sola pregunta 
de si se hace o no ejercicio, o la suposición de un nivel de actividad física en relación con la profesión del sujeto, no se ha probado como suficientemente exacto. Es útil también, documentar la sensación subjetiva que deja el trabajo realizado, usando escalas como la de Borg, que está graduada del 1 al 10 y en la que el grado 1 equivaldría a no tener ninguna sensación de haber realizado esfuerzo y el grado 10 correspondería a la percepción de una disnea extrema (71-78).

En nuestros días está apareciendo la figura del llamado entrenador personal, profesional que a manera de testigo permanente vigila y orienta, no solamente sobre las condiciones del ejercicio a realizar por la persona a su cargo, sino que también le aconseja sobre los usos dietéticos concretos a seguir.

\section{CARACTERÍSTICAS PRÁCTICAS DEL EJERCICIO}

Los efectos beneficiosos de la actividad física van a depender de los llamados principios de entrenamiento: De la frecuencia, de la intensidad y del tiempo (17). Respecto a la intensidad con que se realice el trabajo, se recomienda que todos las actividades se desarrollen con una intensidad mediaalta, de forma vigorosa (entendiéndose por ello la cantidad de esfuerzo que produzca la aparición de sudoración y taquicardia, o bien que suponga la realización de un trabajo mayor de 4 METS, o que lleve al desarrollo de un ejercicio a un 50\% del nivel máximo de consumo de oxígeno). Sin embargo, esta actividad, no debe ser extenuante (79). El cómputo del tiempo empleado se deberá ajustar proporcionalmente a la intensidad del ejercicio, así, 30 minutos de marcha equivaldrán a 15 minutos de carrera.

El tipo de ejercicio debe ser tenido en cuenta. Si éste se efectúa en recorrido llano o en pendiente. Una inclinación ascendente de un $10 \%$ a igualdad de ejercicio, dobla la intensidad del mismo. Por ejemplo la marcha en llano equivale a 4 METS, mientras que si se realiza en una pendiente ascendente del $10 \%$ suponen un incremento hasta 8 METS. El esfuerzo realizado varía también según los grupos musculares implicados, de esa forma, al nadar se utilizan los cuatro miembros, por ello supone mayor gasto de energía, 8 METS. El esfuerzo de subir escaleras lleva una intensidad de 8 METS, si no se utiliza la barandilla para apoyarse.

Cuando se comentan los efectos beneficiosos de la actividad física siempre se acentúa la importancia de su componente aerobio. Sin embargo, es inevitable, que durante cualquier trabajo, se realicen algunos ejercicios con componente anaerobio. El ejercicio anaerobio es recomendable cuando se quiere promover cierto grado de desarrollo muscular, lo que en algunos casos es beneficioso. Por ejemplo en las personas ancianas o en enfermos emaciados (80). Igualmente parece aconsejable incluir el uso de movimientos que faciliten la flexibilidad articular y que tonifiquen y equilibren la dinámica del conjunto motor. Se ha comprobado no obstante que la realización aislada de ejercicios de flexibilidad y de relajación, al consumir poca energía, no aportan ventajas a los efectos metabolicos que se persiguen. En este contexto, no son recomendables los ejercicios culturistas en los que se extreman la anaerobiosis y flexibilidad, para llevar a un desarrollo considerable de la masa muscular, aún a costa a veces, del riesgo de provocar rhabdomiolisis y/o hipertensión arterial.

La actividad física tiene los máximos efectos coste/beneficio cuando se lleva acabo dentro de una banda de intensidades determinada. Por encima y por debajo de unos límites no se obtiene, de forma importante, una aumento de los resultados deseados (81). Esto ocurre con ciertas actividades poco intensas y que no llegan a producir un efecto, cómo por ejemplo: En el trabajo de ama de casa, con poca familia y hogar muy mecanizado. Si se juega al golf con coche eléctrico. Al realizar paseos reposados y tranquilos. Por el contrario, en el otro extremo, una actividad física muy intensa tampoco mejora claramente los resultados saludables, como sería el caso de correr más de $50 \mathrm{~km}$ a la semana (82).

Se debe tener en cuenta que los valores que se comentan son orientadores. Las cifras muestran ligeras variaciones entre autores. Están referidas al individuo estándar de $70 \mathrm{Kg}$ de peso y 1,70 m de altura. La variación de los parámetros personales modificaran proporcionalmente el esfuerzo a realizar.

\section{RECOMENDACIONES}

El aumento del trabajo físico puede realizarse de dos maneras, bien mediante aumento de la actividad física diaria, con modificaciones puntuales y acumulables de la conducta individual, sin dedicarle un tiempo extra (83), o bien mediante la práctica de ejercicio físico regular y fuera de la vida diaria por medio de deporte, juegos y gimnasios.

La actividad física recomendada debe ser fundamentalmente aeróbica (marcha, correr, nadar, bicicleta, subir y bajar escaleras). Debe iniciarse progresivamente para lograr un entrenamiento, a partir de la actividad habitual que se realizara con anterioridad. La meta a alcanzar será una actividad o ejercicio moderado, de al menos 4 METS. El gasto calórico deberá aproximarse a las 1.000-4.000 Kcal a la semana, o a los 6.000-8.000 Kjul a la semana (72). El movimiento resultante, globalmente considerado, deberá producir más de 10.000 pasos al día. El tiempo empleado será de más de 30 minutos al día, hasta llegar a los 60 , tomando como referencia intensidades de ejercicio de 4 METS. La frecuencia de realización será de más de 3 días a la semana, hasta alcanzar los 5 días. Si se utilizan escaleras, se subirán alrededor de 33 pisos a la semana, hasta unos 50 . Se puede considerar que un piso suele tener dos o tres tramos de escalera y un tramo suele tener 8 escalones, cada escalón mide unos $20 \mathrm{~cm}$ de altura (84-90).

Se buscarán actividades participativas. Juegos, excursiones.

Los efectos del ejercicio físico son acumulables, si éste se desarrolla en pequeños periodos de tiempo a lo largo del día. No es necesario efectuarlo de una sola vez (91).

Debe evitarse la realización de ejercicio sólo durante los fines de semana, si en los días laborables se lleva una vida sedentaria. Lo que se ha llamado el síndrome del "weekend warrior" (92). Para conseguir una actividad cuantitativamente beneficiosa, al ser esta obligadamente concentrada, exigiría desarrollar demasiado esfuerzo de una vez, que podría ser peligroso.

Si se quiere intervenir sobre la actividad física sin dedicar un tiempo extra se pueden utilizar procedimientos que modifiquen el comportamiento individual diario, aplicando alguno de los siguientes recursos:

1. En el trabajo: caminar más al ir al trabajo. Bajar y subir del/al autobús una o dos parada antes o después. Dejar el coche aparcado a uno o dos kilómetros de distancia, si es que 
se dispone de aparcamiento en las proximidades. Hablar por teléfono de pie. Aprovechar la pausa en el trabajo para dar una vuelta a la manzana o inmueble. Las sesiones de trabajo (Brainstorm) entre dos o tres colegas se harán caminando. Nunca coger ascensor para subir uno/dos pisos o bajar dos/tres pisos. En distancias cortas ir a hablar personal y directamente con el interlocutor sin usar el teléfono. Recoger inmediatamente del suelo lo que se caiga. Caminar en las esperas de los aeropuertos.

2. En el ocio se pueden propiciar actividades de desarrollo físico: baile (clásico: 3 METS, moderno: 8 METS), deportes, juegos, sociedades deportivas, clubs (el ski alpino y el tenis: unos 8 METS, golf andando: 4 METS, golf en coche eléctrico: 2 METS).

\section{PRECAUCIONES}

Si se tienen más de 40 años siendo varón o más de 50 años para el sexo femenino, se padece alguna enfermedad o se presentan síntomas en reposo o en el ejercicio cómo dolor, disnea, mareos o pérdidas de conciencia, el sujeto deberá ser sometido al correspondiente examen médico, que dictamine sobre la idoneidad de dicha esfuerzo. La actividad física debe suspenderse ante toda situación patológica aguda, como insuficiencia cardiaca, descompensación diabética, insuficiencia coronaria, etc, pues en estos casos sería contraproducente y constituiría un agravante.

Las contraindicaciones para hacer ejercicio físico son las siguientes: a) Absolutas: infarto agudo de miocardio en los 3 y 5 primeros días. Angina inestable. Arritmias sintomáticas no controlables. Endocarditis activa. Estenosis aórtica severa sintomática. Insuficiencia cardiaca sintomática no controlable. Embolia o infarto pulmonar agudo. Infección. Insuficiencia renal, Tirotoxicosis, Pericarditis y Miocarditis agudas; b) relativas: trombosis de extremidades inferiores, hipertensión arterial o pulmonar intensa. Miocardiopatía hipertrófica. Alteraciones mentales que impiden la colaboración. Alteraciones electrolíticas. Estenosis valvular cardiaca moderada, Estenosis del tronco izdo coronario.

\section{MEDIDAS SOCIALES Y COMUNITARIAS}

Todas las normas que se dan sobre conductas sanitarias generales, bien traten sobre la actividad física o los regímenes alimenticios, encuentran la dificultad del cumplimiento a medio y largo plazo, y no solamente en aquellas situaciones en las que se recomienda para efectuar una prevención primaria de una dolencia concreta, sino también cuando se utilizan para la prevención secundaria, inclusive cuando tiene implicaciones curativas inmediatas. Es necesario que las normas sean personalizadas, simples y claras, para que se entiendan y se acepten. Los conceptos fundamentales deben saberse individualizar en cada caso concreto, de acuerdo con las características y circunstancias de la persona. Es imprescindible, la adopción de medidas que animen y propicien estas conductas proclives a la actividad física, por parte de las instituciones sociales, como son las organizaciones sanitarias, las educativas y comunitarias, creando, publicitando e incentivando unos modelos de pautas beneficiosas en la población. No será fácil, se tendrá que intervenir contra comportamientos sociales muy arraigados o sobre usos que proporcionan un bienestar, mal entendido. Se tendrán que combatir filosofías economicistas-consumistas, como son los excesos en la utilización del coche, del ascensor, del teléfono, de la televisión, del mando a distancia, del móvil, etc. En la bibliografía se han visto analizadas minuciosamente hasta los procedimientos de propaganda a emplear. Es interesante conocer sobre la oportunidad y efectividad que las medidas puedan tener, dependiendo de la localización, tamaño y extensión de los mensajes en los letreros de aviso sobre el beneficio del ejercicio físico, invitando a utilizar escaleras, prescindir del ascensor, etc. Igualmente se ha constatado sobre la poca efectividad de los cursos teóricos en torno a la divulgación de la importancia del ejercicio físico, si no van seguidos de actividades que creen actitudes (93). En este sentido diversos autores acentúan la necesidad de crear ambientes propicios, en la forma de instalaciones urbanas abiertas, que hagan agradable y accesible la realización de ejercicio físico (94).

\section{Bibliografía}

1. Eden KB, Tracy Orleans C, Mulrow CD, Pender NJ, Teutsch SM. Does Counseling by Clinicians Improve Physical Activity? A Summary of the Evidence for the U.S. Preventive Services Task Force. Annals of Internal Medicine 2002;137: 208-215.

2. Pate RR, Pratt M, Blair SN, Haskell WL, Macera CA, Bouchard C, Buchner D, Ettinger W, Heath GW, King AC, Kriska A, Leon AS, Marcus BH, Morris J, Paffenbarger RS, Patrick K, Pollock ML, Rippe JM, Sallis J, Wilmore JH. Physical Activity and Public Health: A Recommendation From the Centers for Disease Control and Prevention and the American College of Sports Medicine.[Special Communication]. JAMA $1995 ; 273: 402-7$.

3. Saltin B, H Pilegaard: Metabolic fitness: Physical activity and health. Ugeskr Laeger 2002;164:2156-62.

4. Cinquegrana G, L Spinelli, L D’Aniello, M Landi, MT D’Aniello, P Meccariello. Exercise training improves diastolic perfusion time in patients with coronary artery disease. Heart Dis 2002; 4: 13-7.
5. Houston TK, Meoni LA, Ford DE, Brancati FL, Cooper LA, Levine DM, Liang KY, Klag MJ. Sports ability in young men and the incidence of cardiovascular disease. Am J Med 2002 Jun 15; 112: 733-4.

6. Leon AS.Physical activity levels and coronary heart disease. Analysis of epidemiologic and supporting studies. Med Clin North Am 1985; 69: 3-20.

7. Norris JN, Clayton DG, Everitt MG, et al: Exercise in leisure time:coronary attack and death rates. Br Heart J 1990; 63: 325-334.

8. Akashi YJ, Koike A, Osada N, Omiya K, Itoh H. Short-term physical training improves vasodilatory capacity in cardiac patients. Jpn Heart J 2002; 43: 13-24.

9. Adamopoulos S, J Parissis, D Karatzas, C Kroupis, M Georgiadis, G Karavolias, J Paraskevaidis, K Konavitou, AJ Coats, DT Kremastinos. Physical training modulates proinflamatory citokines and the soluble Fas/soluble Fas ligand system in patients with chronic heart failure. J Am Coll Cardiol 2002; 39: 653-63. 
10. Rheaume C, PH Waib, Y Lacourciere, A Nadeau, J Cleroux: Efectos del ejercicio ligero para la sensibilidad a la insulina en enfermos hipertensos. Hypertension, 2002; 39: 989-95.

11. Lee CD, Blair SN. Cardiorespiratory fitness and stroke mortality in men. Med Sci Sports Exerc 2002; 34: 592-552.

12. Kirk AF, LA Higgins, AR Hughes, BM Fisher, N Mutrie, S Hillis, PD MacIntyre. A randomized, controlled trial to study the effect of exercise consultation on the promotion of physical activity in people with Type 2 diabetes: a pilot study. Diabet Med 2001; 18: 877-82.

13. Karlsson MK: Skeletal effects of exercise in men. Calcif Tissue Int 2001; 69: 196-9.

14. Venth RT. Role of physical activity for the prevention and rehabilitation of osteoporosis. Z Gastroenterol 2002, 40 (Supl. 1): 62-7.

15. Fairey AS, KS Courneys, CJ Field, JR Mackey. Physical exercise and immune system function in cáncer survivors a comprehensive review and future directions. cáncer 2002; 94: 539-51.

16. Wannamethee SG, AG Shaper, M Walker. Physical activity and risk of cáncer in middle-aged men. Br J cáncer 2001; 85: 1311-6.

17. American College of Sports Medicine. The recommended quantity and quality of exercise for developing and maintaining cardiorespiratory and muscular fitness in healthy adults.Med Sci Sports Exerc 1990;22:265-74.

18. Durstine JL, Grandjean PW, Davis PG, Ferguson MA, Alderson NL, DuBose KD. Blood lipid and lipoprotein adaptations to exercise: a quantitative analysis. Sports Med 2001; 31: 1033-62.

19. Fahlman MM, Boardley D, Lambert CP, Flynn MG. Effects of endurance training and resistance training on plasma lipoprotein profiles in elderly women. : J Gerontol A Biol Sci Med Sci 2002 ;57: B54-60.

20. Fujino Y, T Mizoue, N Tokui, T Yoshimura. Walking exercise and its relationship to serum lipids Japanese. J Epidemiol 2002; 12: 64-9.

21. Higuchi M, Iwaoka K, Fuchi T, et al: Relation of running distance to plasma HDL-cholesterol level in middle-aged male runners. Clin Physiol 1989; 9: 121-130.

22. Kostka T, JR Lacour, M Bonnefoy. Response of blood lipids to physical exercise in elderly subjects. Prev Cardiol 2001; 4: 122-125.

23. Senti M, Elosua R, Tomas M, Sala J, Masia R, Ordovas JM, Shen H, Marrugat J. Physical activity modulates the combined effect of a common variant of the lipoprotein lipase gene and smoking on serum triglyceride levels and high-density lipoprotein cholesterol in men. : Hum Genet 2001; 109: 385-92.

24. Van der Eems K, AH Ismail. Serum lipids: Interaction between age and moderate intensity exercise. Br J Sports Med 1985; 19: 112-4.

25. Kokkinos PF, Holland JC, Narayan P. Miles run per week and highdensity lipoprotein cholesterol levels in healthy, middle-aged men: a dose-response relationship. Arch Intern Med 1995; 155: 415-420.

26. Kraemer RR, EO Acevedo, LB Synovitz, RJ Duran, LG Johnoson, E Petrella, MS Fineman, T Gimpel, VD Castracane. Glucoregulatory endocrine response tom intermittent exercise of different intensities: plasma changes in a pancreatic beta-cell peptide, amylin. Metabolism 2002; 51: 657-63.

27. Lehmann R, Engler H, Honegger R, Riesen W, Spinas GA. Alterations of lipolytic enzymes and high-density lipoprotein subfractions induced by physical activity in type 2 diabetes mellitus. Eur J Clin Invest 2001; 31: 37-44.

28. Derouich M, Boutayeb A. The effect of physical exercise on the dynamics of glucose and insulin. J Biochem 2002; 36: 911-7

29. Abramson JL, V Vaccarino. Relationship between physical activity and inflammation among apparently healthy middle-aged and older US adults. Arch Intern Med 2002; 162:1286-92.

30. Simonson SR: The immune response to resistance exercise. J Strength Cond Res 2001; 15: 378-84

31. Togo M, Y Hashimoto, A Futamura, K Tsukamoto, H Satoh, M Hara, T Watanabe, H Nakarai, K Nakahara, S Kimura. Relationship between the serum level of leptin and life-style habits in japanese men. Res 2000; 54: 169-73.

32. Chen J, WJ Millar. Health effects of physical activity. Health Rep 1999; 11: 21-30.

33. Hale BS, KR Koch, JS Raglin. State anxiety responses to 60 minutes of cross training. Br J Sports Med 2002; 36: 105-7

34. Lee IM, Skerrett PJ. Physical activity and all-cause mortality: what is the dose-response relation?. Med Sci Sports Exerc 2001;33:S459-71.

35. Trost SG, RR Pate, JF Sallis, PS Freedson, WC Taylor, M Dowda, J Sirad. Age and gender differences in objetively measured physical activity in youth. Med Sci Sports Exerc 2002; 34: 350-5.

36. Norman A, Bellocco R, Vaida F, Wolk A. Total physical activity in relation to age, body mass, health and other factors in a cohort of Swedish men. Int J Obes Relat Metab Disord $2002 ; 26: 670-5$

37. Ford BS, McDonald TE, Owens AS, Robinson TN. Primary care interventions to reduce television viewing in African-American children Am J Prev Med 2002; 22: 106-9.

38. Seefeldt V, RM Malina, MA Clark: Factors affecting levels of physical activity in adults. Sports Med 2002; 32: 143-68.

39. Sirard JR, RR Pate: Physical activity assessment in children and adolescents. Sports Med 2001; 31: 439-54

40. Kramer JM, JA Beatty, ED Plowey, Waldrop TG. Exercise and hypertension: a model for central neural plasticity. Clin Exp Pharmacol Physiol 2002; 29: 122-6.

41. Borg P, Kukkonen-Harjula K, Fogelholm M, Pasanen M. Effects of walking or resistance training on weight loss maintenance in obese, middle-aged men: a randomized trial. Int $\mathbf{J}$ Obes Relat Metab Disord 2002; 26: 676-83.

42. Long SJ, K Hart, LM Morgan: The ability of habitual exercise to influence appetite and food intake in response to high- and low-energy preloads in man. Br J Nutr 2002; 87: 517-23.

43. Luke A, RA Durazo-Arvizu, CM Rotini, H Iams, DA Schoeller, AA Adeyemo: Activity energy expediture and adiposity among black adults in Nigeria and the United States. Am J Nclin Nutr 2002; 75; 1045-50.

44. Stromme SB, Hostmark AT. Physical activity overweight and obesity. Tidsskr Nor Laegeforen 2000; 120: 3578-82.

45. Williams PT. Health effects resulting from exercise versus those from body fat loss. Med Sci Sports Exerc 2001; 33 (Supl. 6): S611-21.

46. Roubenoff R, H Schmitz, L Barrios, J Layne, E Potts, GJ Cloutier, F Denry. Reduction of abdominal obesity in lipodystrophy associated with human immunodeficiency virus infection by means of diet and exercise: case report and proof of principle. Clin Infect Dis 2002; 34: 390-3.

47. Kanaley JA, Sames C, Swisher L, Swick AG, Ploutz-Snyder LL, Steppan CM, Sagendorf KS, Feiglin D, Jaynes EB, Meyer RA, Weinstock RS. Abdominal fat distribution in pre- and postmenopausal women: The impact of physical activity, age, and menopausal status. Metabolism 2001; 50: 976-82.

48. McKechnie R, Rubenfire M, Mosca L. Association between self-reported physical activity and vascular reactivity in postmenopausal women. Atherosclerosis 2001; 159: 483-90.

49. Boyden TW, Pamenter RW, Going SB, Lohman TG, Hall MC, Houtkooper LB, Bunt JC, Ritenbaugh C, Aickin M. Resistance exercise training is associated with decreases in serum low-density lipoprotein cholesterol levels in premenopausal women. Arch Intern Med 1993; 153: 97-100.

50. Nieman DC. Exercise immunology: nutritional countermeasures. Can J Appl Physiol 2001; 26: S45-55.

51. Wannamethee SG, GD Lowe, PH Whincup, A Rumley, M Walker, L Lennon. Physical activity and hemostatic and inflamatory variables in elderly men. Circulation 2002; 105: 1785-90.

52. Ji LL. Exercise-induced modulation of antioxidant defense. Ann N Y Acad Sci 2002; 959: 82-92.

53. Sakamoto K, Goodyear LJ. Intracellular signaling in contracting skeletal muscle. J Appl Physiol 2002; 93: 369-383.

54. Bouchard C, Rankinen T. Individual differences in response to regular physical activity. Med Sci Sports Exerc 2001; 33 (Supl. 6): S446-51.

55. Kahara T, T Takamura, T Hayakawa, Y Nagai, H Yamaguchi, T Katsuki. Prediction of exercise-mediated changes in metabolic markers by gene polymorphism. Diabetes Res Clin Pract 2002; 57: 105-10.

56. Rice T, JP Despres, L Perusse, Y Honng, MA Province, J Bergeron: Familial aggregation of blood lipids response to exercise training in the health, risk factors, exercise training, and genetics (HERITAGE) Family Study. Circulation 2002; 105: 1904-8.

57. Schmitz KH, Schreiner PJ, Jacobs DR, Leon AS, Liu K, Howard B, Sternfeld B. Independent and interactive effects of apolipoprotein E phenotype and cardiorespiratory fitness on plasma lipids. Ann Epidemiol 2001; 11: 94-103

58. D'Eon T, B Braun. The roles of estrogen and progesterone in regulating carbohydrate and fat utilization at rest and during exercise. J Womens Health Gend Based Med 2002; 11: 225-37.

59. Steffensen CH, Roepstorff C, Madsen M, Kiens B. Myocellular triacylglycerol breakdown in females but not in males during exercise. Am J Physiol Endocrinol Metab 2002; 282: E634-42

60. Williams PT, PD Wood, WL Haskell. The effects of running milleage and duration on plasma lipoprotein levels. JAMA 1982; 247: 2674-9. 
61. Mazic S, Zivotic-Vanovic M, Igracki I, Zivanic S, Velkovski S. A simple and reliable step-test for indirect evaluation of aerobic capacity. Med Pregl 2001; 54: 522-9.

62. Bassett DR Jr, Ainsworth BE, Swartz AM, Strath SJ, O'Brien WL, King GA. Validity of four motin sensors in measuring moderate intensity physical activity. Med Sci Sports Exerc 2000; 32 (Supl. 2): S47180 .

63. Irimagawa S, Imamiya S. Industrial hygienic study on nursing activities comparison of energy expenditure between pedometer and Holter electrocardiograph. : Kitasato Arch Exp Med 1993; 65: 99-105.

64. Iwane M, Arita M, Tomimoto S, Satani O, Matsumoto M, Miyashita K, Nishio I. Walking 10,000 steps/day or more reduces blood pressure and sympathetic nerve activity in mild essential hypertension. Hypertens Res 2000; 23: 573-80.

65. Schutz Y, S Weinsier, P Terrier, D Durrer. A new accelerometric method to assess the daily walking practice. Ins J Obes Relat Metab Disord 2002; 26: 111-8.

66. Tudor-Locke CE, Bell RC, Myers AM, Harris SB, Lauzon N, Rodger NW. Pedometer-determined ambulatory activity in individuals with type 2 diabetes. Diabetes Res Clin Pract 2002; 55: 191-9.

67. Freedson PS, Miller K. Objective monitoring of physical activity using motion sensors and heart rate. Res Q Exerc Sport 2000; 71: S21-9.

68. Strath SJ, Basselt DR Jr, Swartz AM, Thompson DL. Simultaneous heart rate-motion sensor tchnique to estimate energy expediture. Med Sci Sports Exerc 2001; 33: 2118-23

69. Terrier P, Ladetto Q, Merminod B, Schutz Y. Measurement of the mechanical power of walking by satellite positioning system (PGS). Med Sci Sports Exerc 2001; 33: 1912-8

70. Terrier P, Aminian K, Schutz Y. Can accelerometry accurately predict the energy cost of uphill/downhill walking? Ergonomics 2001 Jan 15;44:48-62.

71. Borg GA. Scale for Rating Perceived exertion. Med Sci Sports Exerc 1982; 14: 377-387.

72. Bunc V, Z Teply. Evaluation of energy requirements in basic physical activity. Cas Lek Cesk 1989; 128: 1580-3.

73. Capodaglio $\mathrm{P}$. The use of subjective rating of exertion in Ergonomics Ital Med Lav Ergon 2002;24 :84-9.

74. Chasan-Taber L, JB Erickson, JW McBride, PC Nasca, S ChasanTaber, PS Freedson. Reproductibility of a self-administered lifetime physical activity questionnaire among female college alumnae. Am J Epidemiol 2002; 155: 282-9.

75. de Ridder CM, HC Kemper, MJ Bertens, AC van Gameren, E Ras, J Voogd, HA Delemarre van der Waal. Concurrent validity of a Weightbearing activity questionnaire in prepuberal and puberal girls and boys. Ann Hum Biol 2002; 29: 237-46.

76. Macera CA, SA Ham, DA Jones, CD Kimsey, BE Ainsworth, LJ Neff: Limitations on the use of a single screening question to measure sedentary behavior. Am J Public Health 2001; 91: 2010-2.

77. Philippaerts RM, KR Westerterp, J Lefevre. Comparison of two ques- tionnarires with tri-axial accelerometer to assess physical activity patterns. Int J Sports Med 2001; 22: 34-9.

78. Sobngwi E, JC Mbana, NC Unwin, TJ Aspray, KG Alberti. Development and validation of a questionnaire for the assessment of physical activity in epidemiological studies in Sub-Saharan Africa. Int J Epidemiol 2001; 30: 1369-70.

79. Smekal G, Pokan R, Baron R, Tschan H, Bachl N. Amount and intensity of physical exercise in primary prevention. Wien Med Wochenschr 2001;151:7-9.

80. Borst SE, Vincent KR, Lowenthal DT, Braith RW. Effects of resistence training on insulin-like growth factor and its binding proteins in men and women aged 60 to 85 . J Am Geriatr Soc 2002; 50: 884-8.

81. Marti B. Health benefits and risks in sports: the other side of the coin. Schweiz Rundsch Med Prax 1989; 78: 290-4.

82. Haennel RG, Lemire F. Physical activity to prevent cardiovascular disease. How much is enough? Can Fam Physician 2002; 48: 67-71.

83. Boreham CA, Wallace WF, Nevill A. Training effects of accumulated daily stair-climbing exercise in previously sedentary young women. Prev Med 2000; 30: 277-81.

84. Howley ET, Colacino DL, Swensen TC. Factors affecting the oxygen cost of stepping on an electronic stepping ergometer. Med Sci Sports Exerc 1992; 24: 1055-1058.

85. Latin RW, Berg K, Kissinger K, Sinnett A, Parks L. The accuracy of the ACSM stair-stepping equation. Med Sci Sports Exerc 2001; 33: 1785-8.

86. Leon AS, Casal D, Jacobs D Jr. Effects of 2,000 kcal per week of walking and stair climbing on physical fitness and risk factors for coronary heart disease. J Cardiopulm Rehabil 1996; 16: 183-92.

87. O'Connell ER, Thomas PC, Cady LD, Karwasky RJ. Energy costs of simulated stair climbing as a job-related task in fire fighting. J Occup Med 1986; 28: 282-4.

88. Shiomi T. Effects of different patterns of stairclimbing on physiological cost and motor efficiency. J Hum Ergol (Tokyo) 1994; 23: 111-20.

89. Teh KC, Aziz AR. Heart rate, oxygen uptake, and energy cost of ascending and descending the stairs. Med Sci Sports Exerc 2002; 34: 695-9:

90. Meyers MC, Sterling JC, Marley RR. Efficacy of Stairclimber Versus Cycle Ergometry in Postoperative Anterior Cruciate Ligament Rehabilitation. Clin J Sport Med 2002; 12; 85-8.

91. Thomas DQ, HL Lewis, ST McCaw, MJ Adams: The effects of continuous and discontinuous walking on physiologic response in collegeage subjects. J Strength Cond Res 2001; 15; 264-5.

92. Linsted KD, Tonstad S, Kusma JW. Self-report of physical activity and patterns of mortality in Seventh-Day Adventist men. J Clin Epidemiol 1991; 44: 355-7.

93. Cardinal BJ, KM Jacques. Evaluation of a university course aimed at promoting exercise behavior. J Sports Med Phys Fitness 2002; 42: 1139 .

94. Giles-Corti B, Donovan RJ. The relative influence of individual, social and physical environment determinants of physical activity. Soc Sci Med 2002; 54: 1793-812. 